\title{
Surveillance of Hepatitis Viruses in Several Small Islands of Japan by Ship: A Public Health Approach for Elimination of Hepatitis Viruses by 2030
}

Tomohiro Akao ${ }^{1}$, Morikazu Onji ${ }^{2}$, Keitarou Kawasaki ${ }^{3}$, Takahide Uehara ${ }^{4}$, Yukima Kuwabara ${ }^{5}$, Takashi Nishimoto ${ }^{6}$, Shinji Yamamoto ${ }^{7}$, Jiro Miyaike ${ }^{8}$, Masaki Oomoto ${ }^{9}$, Teruki Miyake $^{10}$

\begin{abstract}
Aim: In 1990, an epidemiological survey by ship in some Japanese islands revealed high prevalence of hepatitis viruses and human T cell leukemia virus (HTLV). A second prevalence study of these viruses were accomplished in 2018, 28 years after initial survey. Analysis of these studies provide insights about strategies of elimination of hepatitis viruses at remote areas.

Materials and methods: In 2018, prevalence of hepatitis A virus (HAV), hepatitis B virus (HBV), hepatitis C virus (HCV), and HTLV was checked in 305 subjects in the islands those covered in 1990's survey. Hepatitis A virus was checked by the presence of anti-HAV IgG in sera; HBV was affirmed when hepatitis B surface antigen ( $\mathrm{HBsAg}$ ) in sera. Hepatitis C virus infection was evaluated by the presence of antibody to HCV (anti-HCV) and infection with HTLV was estimated by immunoassay. The methodology of assessment of different hepatitis viruses were optimized on the basis of present scientific knowhow. Results: In 1990, the prevalence of HAV (presence of anti-HAV), HBV (presence of HBsAg), HCV (positivity for anti-HCV), and HTLV was found in $79.3 \%, 11.1 \%, 2.2 \%$, and $3.3 \%$ of apparently health subjects of the islands, respectively. The prevalence of HAV, HBV, HCV, and HTLV was $47.9 \%$, $4.6 \%, 1.0 \%$, and $3.0 \%$, respectively, in 2018 . A shift of age of infected persons tilted towards right as the days progressed.

Conclusion: The study indicates a scenario of elimination of hepatitis viruses in Japan as lower trends of prevalence of HAV, HBV, and HCV have been recorded in 2018 compared with 1990, mainly by preventive measures. The most notable finding is that there are almost no new case below the age of 40 years, indicating an effective containment measure against these viruses.

Keywords: Epidemiological survey, Hepatitis viruses, Islands, Japan, Ship-based survey.

Euroasian Journal of Hepato-Gastroenterology (2019): 10.5005/jp-journals-10018-1304
\end{abstract}

\section{INTRODUCTION}

Hepatitis B virus, HCV, and HTLV share the same or similar routes of transmission and infection of these viruses. During and aftermath of II World War, infection of hepatitis viruses progressed considerably. ${ }^{1-4}$ Although there has been distorted socioeconomic state after II World War, Japan is one of the countries that provided due attention to prevent various infectious diseases even during adverse time of their history (when Japan was not a rich country). Ehime prefecture is one of 47 prefectures or regions of Japan and lying at the Western part of Japan. Ehime picture have several islands and proper public health measures are difficult to propagate in these areas. High prevalence of hepatitis and HTLV has been reported from different parts of Ehime prefecture and its Islands. ${ }^{5}$

To assess more insights about prevalence of various infectious diseases, a unique mass screening system has been initiated at Ehime. This has been done by ship so that the remote islands can be enrolled in surveillance process. This is done by a ship named, Saisei Maru (Gift of Social Welfare Organization, Saiseikai Imperial Gift Foundation Inc). The ship is enriched with physicians, as well as instruments so that the surveillance activities can be performed. The ship lands at different remote islands and the inhabitants attend the physicians at Ehime Maru. Using this system, a screening procedure was adapted in 1990 in various islands of Ehime. ${ }^{5}$ Accordingly, several preventive measures were taken to reduce prevalence of these viruses at these areas. Recently, a second surveillance study was accomplished in 2018, about 28 years after the initial survey. The comparative analysis of these two studies would provide insights as to the effect of ship-based surveillance system. The concept of
${ }^{1,3-9}$ Saiseikai Imabari Hospital, Imabari, Ehime, Japan

2,10Ehime University Graduate School of Medicine, Toon, Ehime, Japan

Address reprint requests to: Morikazu Onji, Ehime University Graduate School of Medicine, Toon, Ehime, Japan, Phone: +81 901177 9693, e-mail: morikazu.onji@gmail.com

How to cite this article: Akao T, Onji M, Kawasaki K, et al. Surveillance of Hepatitis Viruses in Several Small Islands of Japan by Ship: A Public Health Approach for Elimination of Hepatitis Viruses by 2030. Euroasian J Hepato-Gastroenterol 2019;9(2):57-62.

Source of support: Nil

Conflict of interest: None

this surveillance system can be adopted for control of hepatitis and other infectious diseases when it is difficult to attend general populations at remote areas.

\section{Materials and Methods}

Saiseimaru, a movable ship, travels periodically in inland Sea of Japan to have insights about public health situations of different islands of Ehime prefecture, Japan. In 2018, during 15 to 18 May, the ship conducted a trip and collected sera from 305 people out of total population of 722 residents in these islands. The study population consisted of 139 men and 166 women. The same ship collected sera from 579 people in 1990. The measurement equipment was ARCHITECTi 2000 SR of Abbott Japan KK, and the reagent was a chemiluminescence immunoassay dedicated 
reagent that was used as the measurement principle. The specimen was stored at $4^{\circ} \mathrm{C}$ until the measurement, and it was measured within 48 hours after the blood collection. There are some differences about the measurement of different viruses in 1990 and 2018. In 1990, anti-IgG antibody was analyzed by commercial kit [anti-HA-lgG antibody: HAAb kit (Dainabbout) (radio immunoassay, RIA)]. Hepatitis $B$ surface antigen was estimated using an assay kit produced by Dianabott (RIA). Anti-HCV was assessed based on presence of $\mathrm{C} 100$ protein [C100-3 antibody kit (Ortho) (enzyme-linked immunosorbent assay)]. Anti-HTLV-I antibody was estimated by Karpas ATLA kit (Denka Seken, enzyme immunoassay)..$^{5}$ Standard control, both positive and negative were used for assays. In 2018, all assays were accomplished based on standard estimation procedures of Japan, ${ }^{6,7}$ whose sensitivity and specificity have been considerable increased over these three decades. This study was approved by Saiseikai Imabari Hospital Ethics Committee (approval number I 29-18) held on September 4, 2017. It was also approved by the Seto Inland Sea Patient Care and Steering Committee (Dr Kazuhide Yamamoto, Chairperson) held on January 15, 2018.

\section{Results}

\section{Altered Population Dynamic in Study Area}

The population of the subject area in 2018 was 722, while it was 1,638 in 1990, the population reduction rate was $55.9 \%$. This is an important part of population dynamic in Japan. There is reduction of population in remote villages and island areas. The people are also getting aged along with time. In 1990, $23.1 \%$ population was aged more than 65 years, whereas, this increased to $44.7 \%$ in 2018 ( $p<0.001$ ). When a comparison was made regarding each group of population, the proportion of population decreased in each age group under the age of 50 years, whereas, those were increased in each group above 60 years (Fig. 1). There was no significant change in male vs female ratio. The proportion of subjects allowed assessment was $42.2 \%$ in 2018 and this was significantly higher than those of 35.3\% in 1990 $(p<0.001)$ indicating the interest of people in these surveys.

\section{Changes of Infection Status}

In 1990, the prevalence of HAV (presence of anti-HAV), HBV (presence of HBsAg), HCV (positivity for anti-C 100), and HTLV was found in $79.3 \%, 11.1 \%, 2.2 \%$, and $3.3 \%$, respectively. The prevalence of these viruses was more in male patients than female patients except the prevalence of HTLV which was a little higher in female patients. The prevalence of HAV, HBV, HCV, and HTLV became $47.9 \%$, $4.6 \%, 1.0 \%$, and $3.0 \%$, respectively, in 2018 (Fig. 2). When these figures were analyzed statistically, it was found that there were no changes in prevalence of HTLV, whereas, the prevalence of HAV and HBV decreased in 2018 compared with those of 1990.

The nationwide prevalence of HAV, HBV, HCV, and HTLV are shown in Figure 3 to develop more insights about the implications of these viruses in these islands. The prevalence of HAV, HBV, and HTLV is significantly higher in these islands compared with those of national average (Fig. 3). However, the ratio of HCV-infected persons was mostly similar to national average (Fig. 3).

Analysis was also accomplished to develop insights about route of infections, as well as to assess whether recent infection has occurred. In 2018, the age of HAV positive patients ranged from
59 years to 93 years; those of HBV positive cases were 47 years to 85 years; HCV positive cases were 59 years to 67 years; and HTLV cases were 56 years to 82 years (Fig. 4).

In 1990, there were HBV-infected persons under the age of 40 years. Also, HCV-, HAV-, and HTLV-infected persons under 50 years old. A shift of these infections with older ages have been documented by this study (Fig. 5).

\section{Discussion}

The study described here have provided important information that can be used for containment of hepatitis viruses in global basis. World Health Organization (WHO) and other affiliated health regulatory international bodies have recently accepted the health and social impacts of infection with different viruses on global basis. Billions of people are infected with these viruses and millions are chronically infected with these viruses. ${ }^{8}$ The chronic infected people act as reservoir of viruses and the virus is transmitted to health individuals from these chronically infected patients who act as permanent and living reservoirs of hepatitis viruses. Also, overall public health status of each country influences infection by hepatitis viruses.

It is well known that HAV causes mainly acute hepatitis and epidemics of HAV is a regular event in many developing countries. The virus is usually transmitted via food and drinks. On the other hand, HBV and HCV induce chronic infections and cause progressive liver diseases, such as liver cirrhosis and hepatocellular carcinoma. ${ }^{9}$ These viruses are transmitted by blood borne method due to transfusion or usage of blood or body fluid from infected persons. The numbers of death due to hepatitis virus have surpassed more than one million. Also, new infection could not be arrested in most developed countries. Several social and economic factors have been cited to be main factors relating to lack of control of hepatitis viruses.

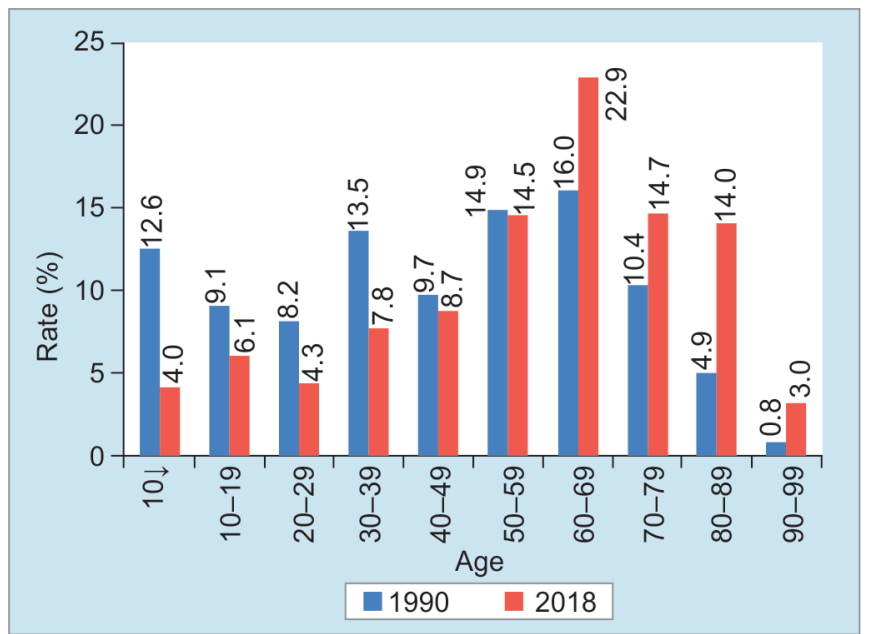

Fig. 1: Comparison of age composition of population. The proportion of each age group in units of 10 years to the population of the examination target area was compared between 1990 and 2018. The population of 1990 is 1638 people, and the population of 2018 is 722 people. The population composition in 2018 peaks at $22.9 \%$ in the age of 60 s, and gradually increases from the age of 10 years old. After that, it is very high, $14.7 \%$ in the 70 s and $14.0 \%$ in the 80 s. Compared with 1990 , the proportion of each age group from the age of 10 s to the age of 50 s decreased, increased in each age group after the age of $60 \mathrm{~s}$, and it was an aging of the examination target area 

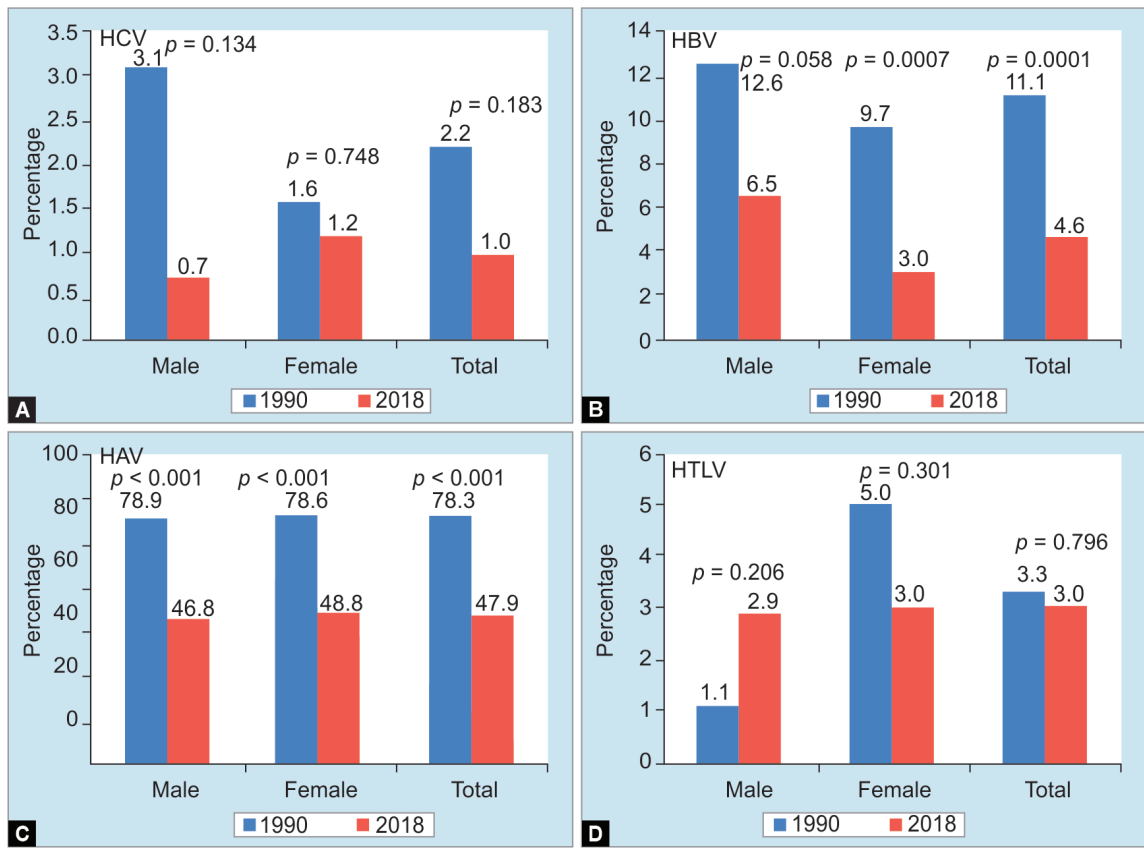

Figs $2 \mathrm{~A}$ to D: Trend of positive rate of each virus infection in 29 years. Positive examples of each virus and positive rate were HCV, 3 cases, $1.0 \%$, hepatitis B virus 14 cases, $4.6 \%$, hepatitis A virus 146 cases, $47.9 \%$, and human T cell leukemia virus 9 cases, $3.0 \%$. As a result of comparing the positive rate of each virus of 29 years, (A) HCV: male patients was $0.7 \%$ from $3.1 \%$, female patients was $1.6 \%-1.2 \%$, total as a whole from 2.2 to $1.0 \%$, and there was no significant difference $(p=0.134, p=0.748, p=0.183)$; (B) Hepatitis B virus: male patients did not differ significantly from 12.6 to $6.5 \%(p=0.058)$. There was a significant difference among female patients from 9.7 to $3.0 \%(p=0.007)$, the total difference was significant from 11.1 to $4.6 \%$ ( $p=0.001)$; (C) Hepatitis A virus: male patients ranged from 78.9 to $46.8 \%$, female patients ranged from 79.6 to $48.8 \%$, total from 79.3 to $47.9 \%$, all differing significantly $(p<0.001)$; (D) Human T cell leukemia virus: there was no significant difference between male patients from 1.1 to $2.9 \%$, female patients from 5.0 to $3.0 \%$, total from 3.3 to $3.0 \%(p=0206, p=0.301, p=0.796)$
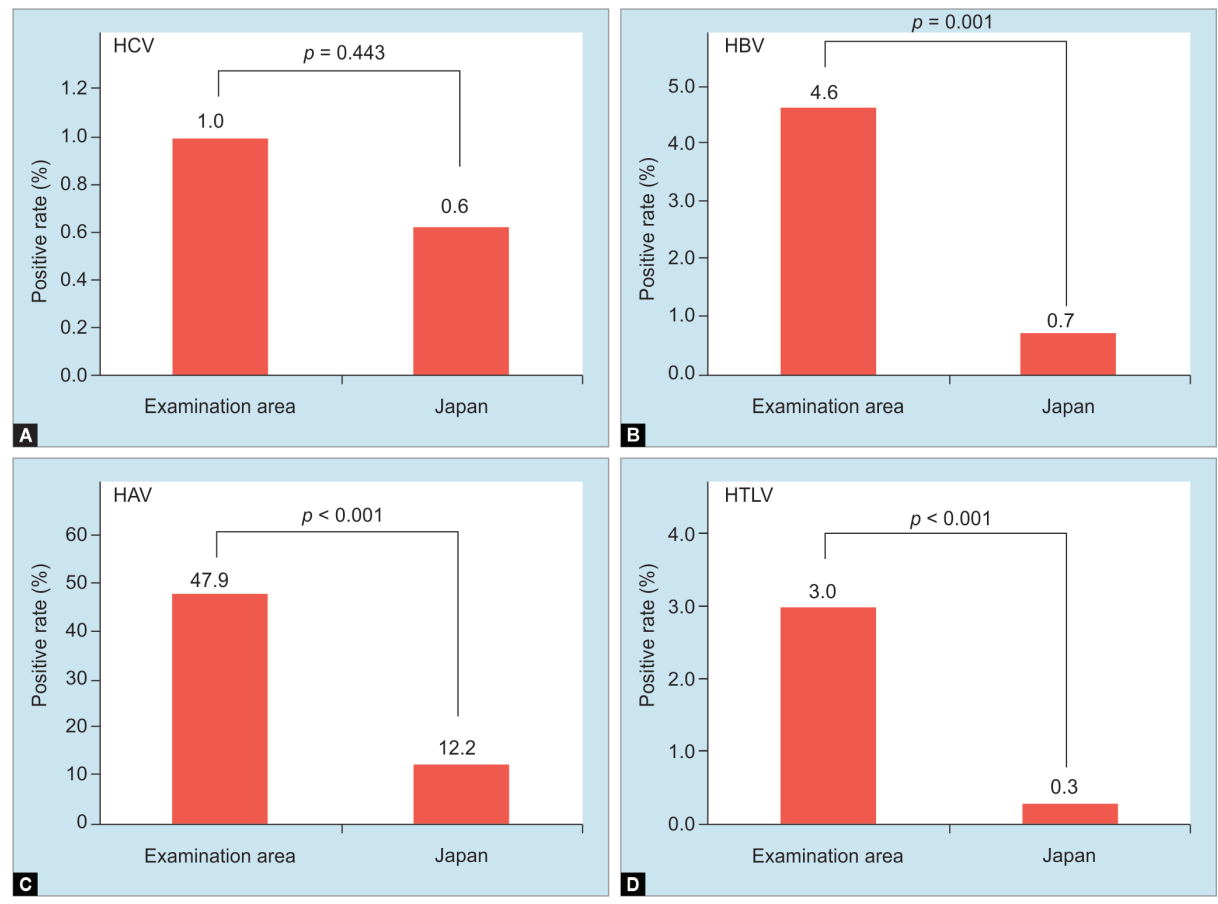

Figs 3A to D: Comparison of each virus infection rate with Japan. The positive rate of each virus in 2018 was compared with the whole country. The following data were used for the nationwide positive rate levels of each virus. HBs antigen and HCV antibody: estimated career data across Japan using donor samples from 2001 to 2006 reported by Tanaka et al. hepatitis A virus antibody: serologic and epidemiologic survey conducted by the National Institute of Infectious Diseases in 2003. Human T cell leukemia virus-1 antibody: survey report of health labor science research team, etc. conducted for donors from 2006 to 2007 . As a result, (A) HCV was $1.0 \%$, which was not significantly different from Japan level $0.6 \%(p=0.443)$; (B) Hepatitis B virus was 4.6\%, which was significantly different from Japan level $0.7 \%(p<0.001)$; (C) The hepatitis A virus was $47.9 \%$, which was significantly different from the Japanese level of $12.2 \%(p<0.001)$; (D) The human T cell leukemia virus was $3.0 \%$, which was significantly different from the Japan level $0.3 \%(p<0.001)$ 

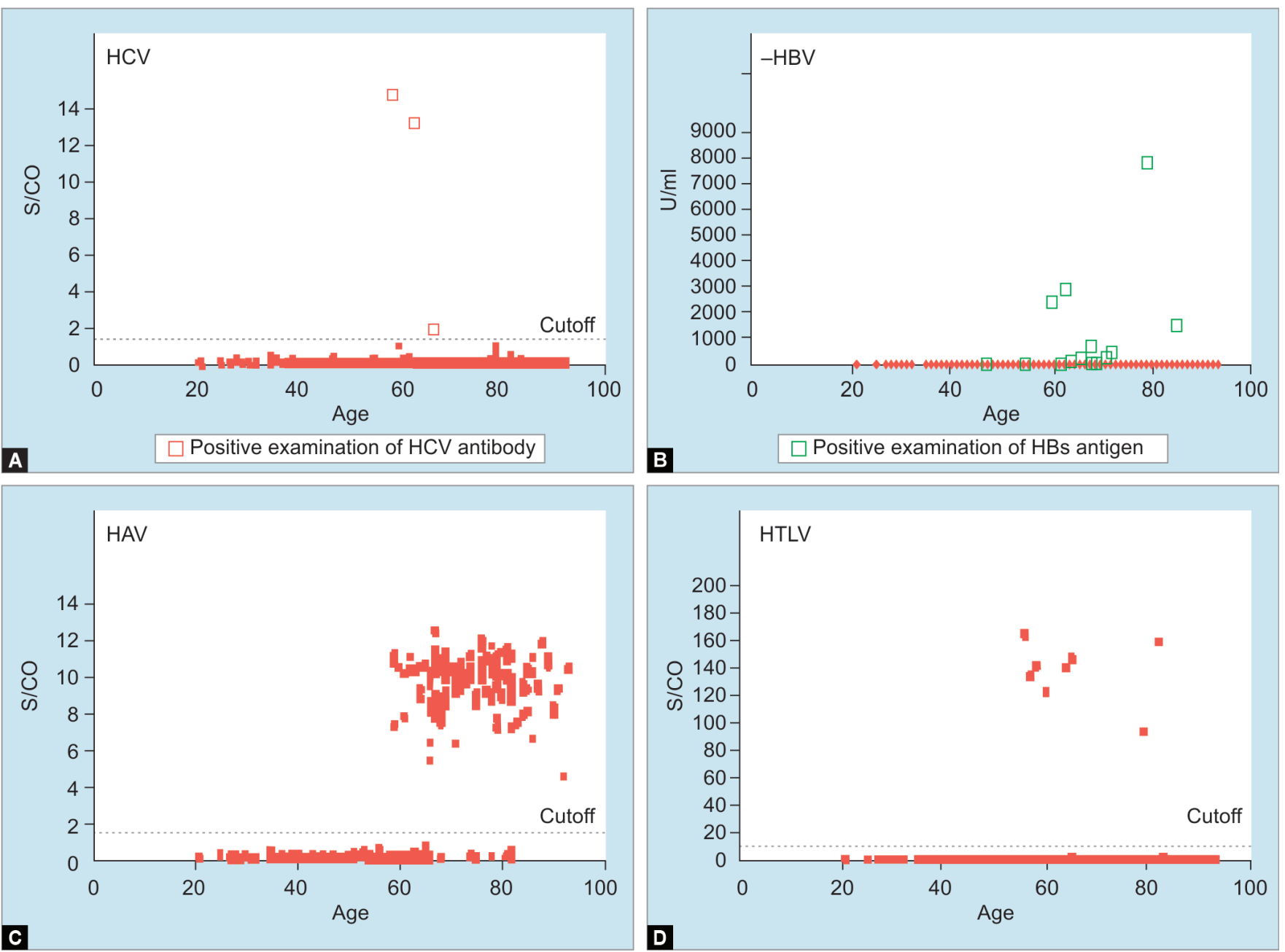

Figs 4A to D: Relationship between each virus infection and age. In 2018, the relationship between HCV, hepatitis A virus, human T cell leukemia virus cutoff index (S/CO), and age and the relationship between $\mathrm{U} / \mathrm{mL}$ of $\mathrm{HBs}$ antigen and age were studied. As a result, the positive of each infection was confirmed in 3 cases of (A) HCV infection 59-67 years old, (B) 14 cases of hepatitis B virus infection 47-85 years old, (C) 146 hepatitis A virus infections 59-93 years old, and (D) 9 cases of human T cell leukemia virus infections 56-82 years old. There were no infected people of less than that age group. In the positive cases near the cutoff, one case of HCV was 1.84 S/CO, three cases of hepatitis B virus were $0.09,0.10$, and $2.91 \mathrm{U} / \mathrm{mL}$. There were no positive cases near the cutoff in hepatitis A virus and human T cell leukemia virus, and all were high titer positive

Japan is one of the developed and advanced countries that harbor quite considerable proportions of different hepatitis viruses. However, Japan initiated a program of control of transmission of hepatitis viruses in mid-1960s and have optimized the surveillance process from time to time. The system that has been shown here represents an exceptional one in which surveillance has been continued via ship to reach to remote areas of certain prefectures. As of today, only $10 \%$ of chronic HBV-infected subjects are aware of their infection status and most of the patients are apparently health and unaware of their infection. One of the major targets of hepatitis elimination by 2030 is to diagnose at least $65 \%$ patients. This can be materialized by unique surveillance system shown here.

Although this study has enrolled population of some islands only and does not represent a nationwide survey of Japan, it shows that neither HAV nor HBV nor HCV have infected any persons below the age of 40 years during last 28 years, although we have not measured anti-HBc in our samples. However, it can be highlighted that there have been no infected patients with hepatitis viruses during last 28 years. This has been possible due to constant surveillance and proper health education advocacy.

Although HCV prevalence has not been significantly reduced, no new infection of HCV could not be substantiated during last 28 years. This bears epidemiologic implication as various measures have blocked new infections with almost all hepatitis viruses. This study also inspired optimism that if hepatitis virus infection can be contained in remote islands, achieving 2030 target of elimination of hepatitis virus (target set by WHO) may not be unachievable goal.

Although reduction of prevalence of different hepatitis virus represents a matter of improved public health situation in Japan, there are areas to be cautious regarding these scenarios at all. Absence of HBV and HCV is always a positive indicator, however reduction of anti-HAV indicates a position of lack of social immunity. 

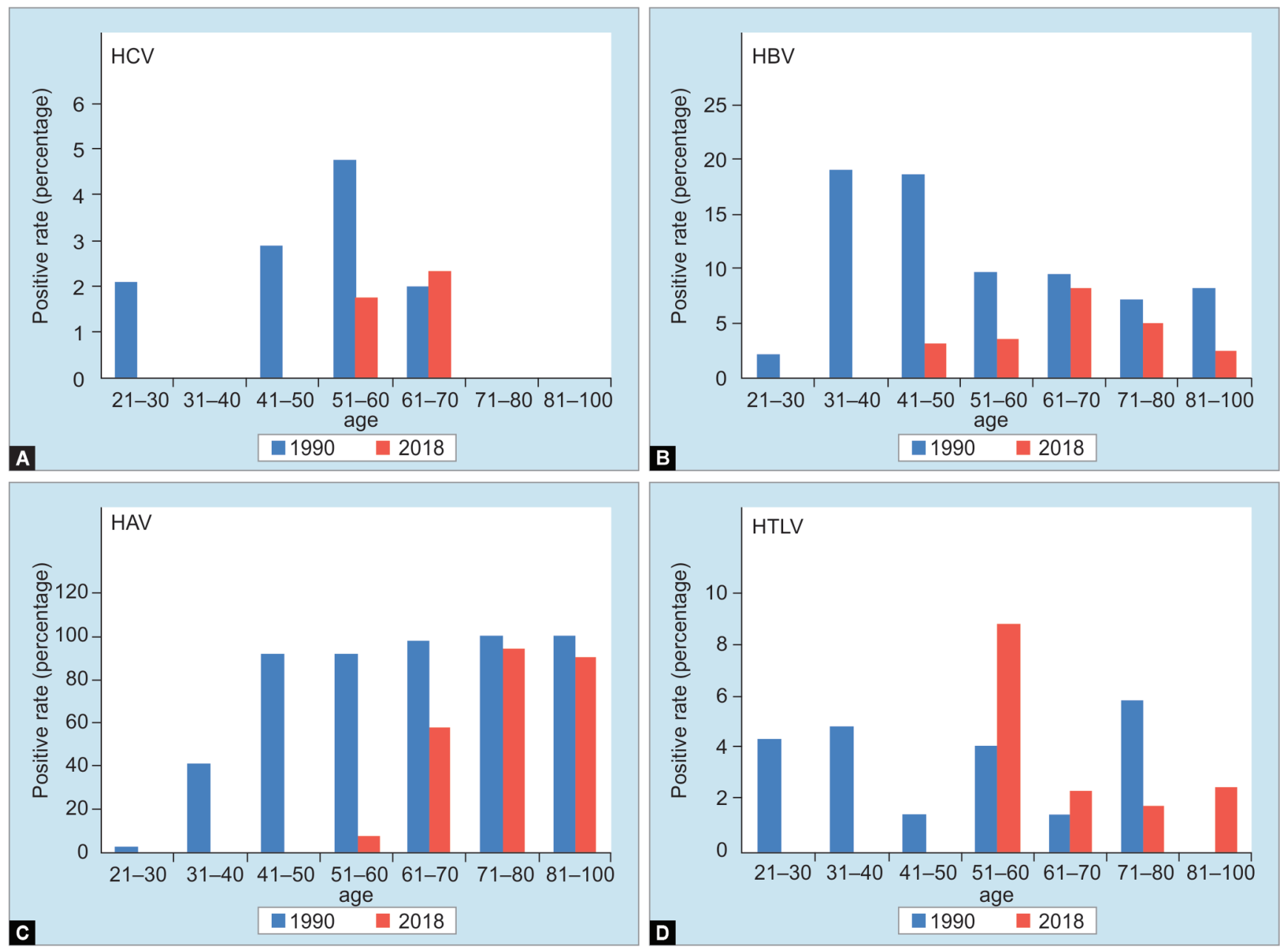

Figs 5A to D: Comparison of positive rate of each virus infection by age group. The infection rates in each age group in 1990 and 2018 were examined. In the 1990 survey, (A) HCV was $2.1 \%$ in the $20 \mathrm{~s}, 2.9 \%$ in the $40 \mathrm{~s}$, (B) hepatitis B virus was $2.1 \%$ in the $20 \mathrm{~s}, 19.0 \%$ in the $30 \mathrm{~s}, 18.6 \%$ in the $40 \mathrm{~s}$, (C) hepatitis A virus was $2.1 \%$ in the $20 \mathrm{~s}, 41.7 \%$ in the $30 \mathrm{~s}, 91.4 \%$ in the $40 \mathrm{~s}$, and (D) human T cell leukemia virus was $4.3 \%$ in the $20 \mathrm{~s}, 4.8 \%$ in the 30 s, and $1.4 \%$ in the $40 \mathrm{~s}$. In the 2018 survey, there were no hepatitis A virus-, HCV-, and human T cell leukemia virus -infected persons under 50 years old, and also were no hepatitis B virus-infected persons under the age of 40 years. The shift of these infections with older ages have been shown in this figure

This may cause sporadic, endemic, and epidemic breakouts of HAV in HAV non-existing areas. ${ }^{10}$ As HAV is a water- and food-borne disease, there should be other measures to counter HAV-induced acute hepatitis. In this study, we also measured antibody to HTLV as this was done before 28 years. It seems that the prevalence of anti-HTLV is not infecting young population.

Taken together, the article indicates the inherent proper surveillance system can downregulate and ultimately eliminate hepatitis viruses in long run.

\section{Acknowledgment}

Authors would like to express their deep appreciation to Dr Tetsuichi Miyashita, Director, Uwajima City National Health Insurance Hiburi Island Clinic for guidance and support for this epidemiologic and clinical investigation and research.

\section{References}

1. Bower WA, Nainan OV, Han X, et al. Duration of viremia in hepatitis A virus infection. J Infect Dis. 2000;182(1):12-17. DOI: 10.1086/315701.
2. Wu JF, Chang MH. Natural history of chronic hepatitis B virus infection from infancy to adult life - the mechanism of inflammation triggering and long-term impacts. J Biomed Sci 2015;22:92. DOI: 10.1186/s12929015-0199-y.

3. Indolfi G, Easterbrook P, Dusheiko G, et al. Hepatitis $C$ virus infection in children and adolescents. Lancet Gastroenterol Hepatol 2019;4(6):477-487. DOI: 10.1016/S2468-1253(19) 30046-9.

4. Fuchi N, Miura K, Tsukiyama T, et al. Natural course of human T-cell leukemia virus type 1 proviral DNA levels in carriers during pregnancy. J Infect Dis 2018;217(9):1383-1389. DOI: 10.1093/infdis/ jiy017.

5. Ochi S, Onji M, Shiraishi K, et al. Prevalence of hepatitis $C$ virus antibody in an area endemic for hepatitis $B$ virus and human $T$ cell leukaemia virus. J Gastroenterol Hepatol 1991;6(6):599-602. DOI: 10.1111/j.1440-1746.1991.tb00918.x.

6. Tanaka J, Koyama T, Mizui M, et al. Total numbers of undiagnosed carriers of hepatitis $C$ and $B$ viruses in Japan estimated by ageand area-specific prevalence on the national scale. Interviology 2011;54(4):185-195. DOI: 10.1159/000324525. 
7. Satake M, Yamaguchi K, Tadokoro K. Current prevalence of HTLV-1 in Japan as determined by screening of blood donors. Med Virol 2012;84(2):327-335. DOI: 10.1002/jmv.23181.

8. Waheed Y, Siddiq M, Jamil Z, et al. Hepatitis elimination by 2030: progress and challenges. World J Gastroenterol 2018;24(44): 4959-4961. DOI: 10.3748/wjg.v24.i44.4959.
9. Kao JH, Chen DS. Global control of hepatitis B virus infection. Lancet Infect Dis 2002;2(7):395-403. DOI: 10.1016/S1473-3099(02) 00315-8.

10. Ishii K, Kiyohara T, Yoshizaki S, et al. Epidemiological and genetic analysis of a 2014 outbreak of hepatitis A in Japan. Vaccine 2015;33(45):6029-6036. DOI: 10.1016/j.vaccine.2015.04.061. 